\title{
Towards Leveraging Backdoors in Qualitative Constraint Networks
}

\author{
Michael Sioutis ${ }^{1[0000-0001-7562-2443]}$ and Tomi Janhunen ${ }^{1,2}[0000-0002-2029-7708]$ \\ 1 Department of Computer Science, Aalto University, Espoo, Finland \\ 2 Tampere University, Tampere, Finland \\ firstname.lastname@aalto.fi
}

\begin{abstract}
In this short paper we introduce the notions of backbones and backdoors in the context of qualitative constraint networks. As motivation for the study of those structures, we argue that they can be used to define collaborative approaches among SAT, CP, and native tools, inspire novel decomposition and parallelization techniques, and lead to the development of adaptive constraint propagators with a better insight into the particularities of real-world datasets than what is possible today.
\end{abstract}

Keywords: Qualitative constraints - Spatio-temporal reasoning - Local consistencies · Backdoors · Backbones.

\section{Introduction}

Qualitative Spatial and Temporal Reasoning (QSTR) is a Symbolic AI approach that deals with the fundamental cognitive concepts of space and time in a qualitative, human-like, manner [12,7]. For instance, in natural language one uses expressions such as inside, before, and north of to spatially or temporally relate one object with another object or oneself, without resorting to providing quantitative information about these entities. QSTR provides a concise framework that allows for rather inexpensive reasoning about entities located in space or time and, hence, further boosts research and applications to a plethora of areas such as dynamic GIS [4], cognitive robotics [8], and deep learning [11,1]. Qualitative spatial or temporal information can be typically captured by a qualitative constraint network (QCN), i.e., a network of constraints corresponding to qualitative spatial or temporal relations between the respective kinds of variables.

Here, we introduce the notions of backbones and backdoors [21] in the context of QSTR, in order to facilitate the integration of QCNs into more generic paradigms such as SAT and $\mathrm{CP}$ on the one hand, but also motivate the further study of QCNs themselves in an effort to obtain a better understanding of their computational characteristics on the other hand. In short, a backbone in a given QCN represents the part of it that can only map to a single qualitative configuration (e.g., the relationship between two regions can only be such that one is contained inside the other), and a backdoor in a given QCN represents its intractable part for some local consistency (i.e., utilizing that consistency alone does not allow one to decide the satisfiability of the QCN). We argue that these notions can drive both theoretical and practical future research (see Section 4). 


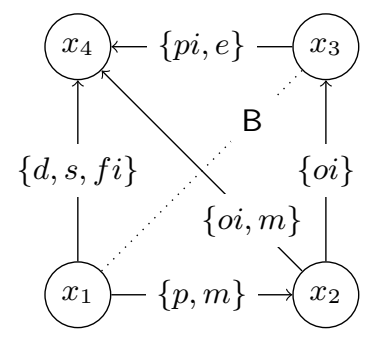

(a) A satisfiable QCN $\mathcal{N}$

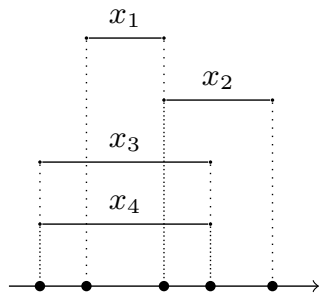

(b) A solution $\sigma$ of $\mathcal{N}$

Fig. 1: Figurative examples of QCN terminology using Interval Algebra; symbols $p, e$, $m, o, d, s$, and $f$ correspond to the atoms precedes, equals, meets, overlaps, during, starts, and finishes respectively, with $\cdot i$ denoting the converse of $\cdot($ note that $e i=e$ )

\section{Preliminaries}

A binary qualitative constraint language is based on a finite set B of jointly exhaustive and pairwise disjoint relations, called the set of base relations [13], that is defined over an infinite domain $D$. These base relations represent definite knowledge between two entities with respect to the level of granularity provided by the domain $D$; indefinite knowledge can be specified by a union of possible base relations, and is represented by the set containing them. The set B contains the identity relation Id, and is closed under the converse operation $\left(^{-1}\right)$. The total set of relations $2^{\mathrm{B}}$ is equipped with the usual set-theoretic operations of union and intersection, the converse operation, and the weak composition operation denoted by $\diamond$ [13]. For all $r \in 2^{\mathrm{B}}, r^{-1}=\bigcup\left\{b^{-1} \mid b \in r\right\}$. The weak composition $(\diamond)$ of two base relations $b, b^{\prime} \in \mathrm{B}$ is defined as the smallest (i.e., strongest) relation $r \in 2^{\mathrm{B}}$ that includes $b \circ b^{\prime}$, or, formally, $b \diamond b^{\prime}=\left\{b^{\prime \prime} \in \mathrm{B} \mid b^{\prime \prime} \cap\left(b \circ b^{\prime}\right) \neq \emptyset\right\}$, where $b \circ b^{\prime}=\left\{(x, y) \in \mathrm{D} \times \mathrm{D} \mid \exists z \in \mathrm{D}\right.$ such that $\left.(x, z) \in b \wedge(z, y) \in b^{\prime}\right\}$ is the (true) composition of $b$ and $b^{\prime}$. For all $r, r^{\prime} \in 2^{\mathrm{B}}, r \diamond r^{\prime}=\bigcup\left\{b \diamond b^{\prime} \mid b \in r, b^{\prime} \in r^{\prime}\right\}$.

As an illustration, consider the well-known qualitative temporal constraint language of Interval Algebra (IA), introduced by Allen in [2]. IA considers time intervals (as temporal entities) and the set of base relations $\mathrm{B}=\{e, p, p i, m$, $m i, o, o i, s, s i, d, d i, f, f i\}$ to encode knowledge about the temporal relations between intervals on the timeline; the symbols are explained in the caption of Figure 1. Specifically, each base relation represents a particular ordering of the four endpoints of two intervals on the timeline, and $e$ is the identity relation Id.

The problem of representing and reasoning about qualitative information can be modeled as a qualitative constraint network, defined in the following manner:

Definition 1. A qualitative constraint network $(\mathrm{QCN})$ is a tuple $(V, C)$ where:

$-V=\left\{v_{1}, \ldots, v_{n}\right\}$ is a non-empty finite set of variables, each representing an entity of an infinite domain $\mathrm{D}$;

- and $C$ is a mapping $C: V \times V \rightarrow 2^{\mathrm{B}}$ such that $C(v, v)=\{\mathrm{Id}\}$ for all $v \in V$ and $C\left(v, v^{\prime}\right)=\left(C\left(v^{\prime}, v\right)\right)^{-1}$ for all $v, v^{\prime} \in V$.

An example of a QCN of IA is shown in Figure 1a; for clarity, converse relations as well as Id loops are not mentioned or shown in the figure. 
Definition 2. Let $\mathcal{N}=(V, C)$ be a $\mathrm{QCN}$, then:

- a solution of $\mathcal{N}$ is a mapping $\sigma: V \rightarrow \mathrm{D}$ such that, $\forall(u, v) \in V \times V$, $\exists b \in C(u, v)$ such that $(\sigma(u), \sigma(v)) \in b$ (see Figure $1 b)$;

- the constraint graph of $\mathcal{N}$ is the graph $(V, E)$ with $\{u, v\} \in E$ iff $C(u, v) \neq \mathrm{B}$;

- given a subset $S \subseteq V \times V$, a refinement $\mathcal{N}_{S}$ of $\mathcal{N}$ with respect to $S$ is a QCN $\left(V, C^{\prime}\right)$ such that $\forall(u, v) \in S$ we have $C^{\prime}(u, v) \subseteq C(u, v)$; if in addition $\left|C^{\prime}(u, v)\right|=1 \forall(u, v) \in S$, then the refinement $\mathcal{N}_{S}$ is called atomic.

Let us further introduce the following operation that substitutes $C\left(v, v^{\prime}\right)$ with $r \in 2^{\mathrm{B}}$ in a given QCN: given a $\mathrm{QCN} \mathcal{N}=(V, C)$ and $v, v^{\prime} \in V$, we have that $\mathcal{N}_{\left[v, v^{\prime}\right] / r}$ with $r \in 2^{\mathrm{B}}$ yields the QCN $\mathcal{N}^{\prime}=\left(V, C^{\prime}\right)$ defined by $C^{\prime}\left(v, v^{\prime}\right)=r$, $C^{\prime}\left(v^{\prime}, v\right)=r^{-1}$ and $\forall\left(u, u^{\prime}\right) \in(V \times V) \backslash\left\{\left(v, v^{\prime}\right),\left(v^{\prime}, v\right)\right\}, C^{\prime}\left(u, u^{\prime}\right)=C\left(u, u^{\prime}\right)$.

\section{Backbones and Backdoors in QCNs}

In this section we introduce the notions of backbones and backdoors in QCNs. In essence, these notions mirror the respective ones defined in [21] for classical (finite-domain) constraint programming; however, in our context the definitions that we provide are quite different from a technical point of view, in that they involve the constraints of QCNs rather than their variables, and the backdoors particularly are tied to local consistencies instead of sub-solvers (cf. [21]).

Given a QCN, a set of constraints (which we simply represent by the set of pairs of variables they constrain) is called a backbone in that QCN, if there is a unique atomic refinement with respect to the constrained pairs of variables such that the QCN is satisfiable. Formally, a backbone is defined as follows:

Definition 3. Given a $\mathrm{QCN} \mathcal{N}=(V, C)$, a subset $S \subseteq V \times V$ is a backbone in $\mathcal{N}$ iff there exists a unique atomic refinement $\mathcal{N}_{S}$ of $\mathcal{N}$ such that $\mathcal{N}_{S}$ is satisfiable.

Clearly, if a $\mathrm{QCN} \mathcal{N}=(V, C)$ has only one solution, then the entire set $V \times V$ is a backbone in and of itself. In addition, every QCN has a unique largest backbone (if any at all). A backbone should be contrasted with a set of frozen constraints, in the sense defined in [6]; in particular, a frozen constraint is a kind of a hard constraint that pertains to the background knowledge of a given problem. For example, a program may need to be compiled before its execution, which would pose a frozen, fixed, constraint that would not be subject to revision.

Next, we introduce the notion of backdoors. As opposed to backbones, backdoors are defined with respect to some (local) consistency; when a backdoor set of constraints in a given QCN is properly instantiated, the consistency can be utilized to decide the satisfiability of that QCN. We view a consistency ${ }_{G}^{\phi}$, where $\phi$ is some operation (such as the weak composition operation) and $G$ a graph, as a predicate on QCNs, i.e., a function that receives an input QCN and returns true or false depending on whether ${ }_{G}^{\phi}$ holds on that QCN or not respectively. Given a consistency ${ }_{G}^{\phi}$, a subset $\mathcal{R} \subseteq 2^{\mathrm{B}}$ is said to be tractable for ${ }_{G}^{\phi}$ if ${ }_{G}^{\phi}$ is complete for deciding the satisfiability of any QCN defined over $\mathcal{R}$ with respect to some graph $G$. We require that a consistency is well-behaving (cf. [5]), as the notion of a backdoor would be overly complicated to define (and utilize) otherwise. 
Definition 4. A consistency ${ }_{G}^{\phi}$ is well-behaving iff for any $\mathrm{QCN} \mathcal{N}=(V, C)$ and any graph $G=(V, E)$ the following properties hold:

- there exists a unique $\subseteq$-maximal ${ }_{G}^{\phi}$-consistent sub-QCN of $\mathcal{N}$, denoted by ${ }_{G}^{\phi}(\mathcal{N})$ and referred to as the ${ }_{G}^{\phi}$-closure of $\mathcal{N}$ with respect to $G$ (Dominance);

$-{ }_{G}^{\phi}(\mathcal{N})$ is equivalent to $\mathcal{N}$ (Equivalence).

Now we are ready to formally define a backdoor, and a stronger variant of it called a strong backdoor as well.

Definition 5. Given a $\mathrm{QCN} \mathcal{N}=(V, C)$, a subset $S \subseteq V \times V$ is a backdoor (resp. strong backdoor) in $\mathcal{N}$ for a well-behaving consistency ${ }_{G}^{\phi}$ iff for some (resp. every) atomic refinement $\mathcal{N}_{S}$ of $\mathcal{N}$ we have that ${ }_{G}^{\phi}\left(\mathcal{N}_{S}\right)$ is defined over a tractable subset of relations $\mathcal{R} \subseteq 2^{\mathrm{B}}$ for ${ }_{G}^{\phi}$.

Additionally, we can identify the notion of a minimal (strong) backdoor, defined as follows:

Definition 6. Given a $\mathrm{QCN} \mathcal{N}=(V, C)$, a backdoor (resp. strong backdoor) $S$ in $\mathcal{N}$ for a well-behaving consistency ${ }_{G}^{\phi}$ is minimal iff there exists no backdoor (resp. strong backdoor) $S^{\prime}$ in $\mathcal{N}$ for ${ }_{G}^{\phi}$ such that $S^{\prime} \subset S$.

Fundamentally, a backdoor in a given instance for some consistency constitutes the hard part of that instance with respect to that consistency, in the sense that, once that part is dealt with, simply enforcing the consistency in the instance allows deciding its satisfiability.

Example. We present here a detailed example that illustrates the aforementioned notions of backbones and backdoors.

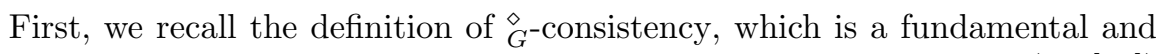
widely used well-behaving local consistency for reasoning with QCNs (cf. [17]) that entails consistency for all triples of variables in a given QCN $\mathcal{N}$ that correspond to three-vertex cycles (triangles) in an accompanying graph $G$.

Definition 7. Given a $\mathrm{QCN} \mathcal{N}=(V, C)$ and a graph $G=(V, E), \mathcal{N}$ is said

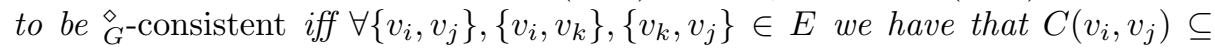
$C\left(v_{i}, v_{k}\right) \diamond C\left(v_{k}, v_{j}\right)$.

If $G$ is the complete graph on the variables of a given QCN, then ${ }_{G}^{\circ}$-consistency becomes identical to $\diamond$-consistency [17], and, hence, $\diamond$-consistency can be seen as a special case of ${ }_{G}^{\circ}$-consistency.

Next, we recall the definition of $\stackrel{\cup}{\cup}$-consistency, which is a well-behaving local consistency that is strictly stronger than any of the known practical consistencies and is based on the idea of partitioning a constraint into singleton relations [20].

Definition 8. Given a $\mathrm{QCN} \mathcal{N}=(V, C)$ and a graph $G=(V, E), \mathcal{N}$ is said to be $\dot{\bullet}_{G}^{\cup}$-consistent iff $\forall\left\{v, v^{\prime}\right\} \in E, \forall b \in C\left(v, v^{\prime}\right)$, and $\forall\left\{u, u^{\prime}\right\} \in E$ we have that

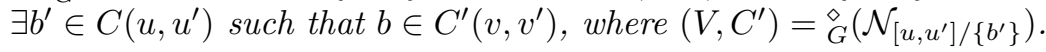




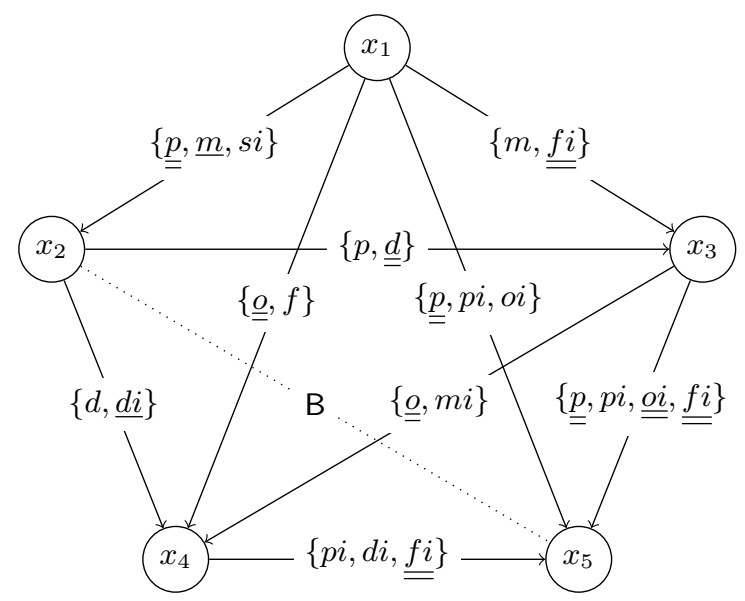

Fig. 2: A QCN $\mathcal{N}$ of Interval Algebra where the underlined base relations are not present in $\left.\stackrel{\diamond}{G}_{(\mathcal{N}}\right)$, and the double-underlined base relations are additionally not present in ${ }_{G}^{\cup}(\mathcal{N}) ; G$ is the constraint graph of $\mathcal{N}$, i.e., the graph that results by removing edge $\left\{x_{2}, x_{5}\right\}$ from the complete graph on $\left\{x_{1}, x_{2}, x_{3}, x_{4}, x_{5}\right\}$, and is chordal

Given either of the above consistencies, the subset $\mathcal{H}_{\mathrm{IA}}$ of the relations of Interval Algebra is tractable for those consistencies; that subset contains exactly those relations that are transformed to propositional Horn formulas when using the propositional encoding of Interval Algebra [16]. Further, that tractability property is maintained with respect to any chordal supergraph of the constraint graph of a given QCN [3].

We proceed with the description of our detailed example. Consider the QCN $\mathcal{N}$ of Interval Algebra shown in Figure 2, along with its accompanying graph $G$ described in the caption. Initially, it can be verified that every constraint that corresponds to some edge in $G$ is defined by a relation that does not belong to the tractable subset of relations $\mathcal{H}_{\mathrm{IA}}$ of Interval Algebra; there are 9 such constraints. After the application of ${ }_{G}$-consistency on $\mathcal{N}$, the relations corresponding to constraints $C\left(x_{1}, x_{2}\right)$ and $C\left(x_{2}, x_{4}\right)$ are refined to $\{p, s i\}$ and $\{d\}$ respectively, with the latter now belonging to $\mathcal{H}_{\mathrm{IA}}$. At this point we can recognize the set $\left\{\left(x_{1}, x_{2}\right),\left(x_{1}, x_{3}\right),\left(x_{1}, x_{4}\right),\left(x_{1}, x_{5}\right),\left(x_{2}, x_{3}\right),\left(x_{3}, x_{4}\right),\left(x_{3}, x_{5}\right),\left(x_{4}, x_{5}\right)\right\}$ as a strong backdoor in $\mathcal{N}$ for ${ }_{G}$-consistency, since any atomic refinement of the corresponding relations will result in $\stackrel{\diamond}{G}_{(}(\mathcal{N})$ being defined over $\mathcal{H}_{\mathrm{IA}}$. Upon closer inspection we can identify the set $\left\{\left(x_{1}, x_{2}\right),\left(x_{4}, x_{5}\right)\right\}$ as a minimal strong back-

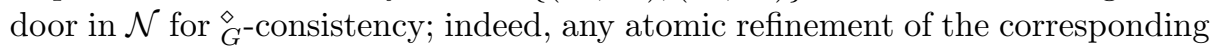
relations will result in $\stackrel{\diamond}{G}(\mathcal{N})$ being defined over $\mathcal{H}_{\mathrm{IA}}$. The singleton $\left\{\left(x_{4}, x_{5}\right)\right\}$ is a backdoor, since there exists an atomic refinement such that ${ }_{G}^{\circ}$-consistency can be used to detect the unsatisfiability of the refined QCN, namely, that of $C\left(x_{4}, x_{5}\right)$ to $\{f i\}$. After the application of ${ }_{G}$-consistency on $\mathcal{N}$, the relations corresponding to constraints $C\left(x_{1}, x_{2}\right), C\left(x_{1}, x_{3}\right), C\left(x_{1}, x_{4}\right), C\left(x_{1}, x_{5}\right), C\left(x_{2}, x_{3}\right), C\left(x_{3}, x_{4}\right)$, $C\left(x_{3}, x_{5}\right)$, and $C\left(x_{4}, x_{5}\right)$ are refined to $\{s i\},\{m\},\{f\},\{p i, o i\},\{p\},\{m i\},\{p i\}$, and $\{p i, d i\}$, respectively, with all relations except the one corresponding to contraint $C\left(x_{4}, x_{5}\right)$ now belonging to $\mathcal{H}_{\mathrm{IA}}$. In fact, at this point we can recognize the 
set $\left\{\left(x_{1}, x_{2}\right),\left(x_{1}, x_{3}\right),\left(x_{1}, x_{4}\right),\left(x_{2}, x_{3}\right),\left(x_{2}, x_{4}\right),\left(x_{3}, x_{4}\right),\left(x_{3}, x_{5}\right)\right\}$ as a backbone in $\mathcal{N}$. Regarding strong backdoors in $\mathcal{N}$ for ${ }_{G}$-consistency, the only choice is the singleton $\left\{\left(x_{4}, x_{5}\right)\right\}$, which is minimal.

In the aforementioned example the use of ${ }_{G}^{\cup}$-consistency made identifying the largest backbone and a minimal strong backdoor in $\mathcal{N}$ for $\stackrel{\leftrightarrow}{G}^{\cup}$-consistency effortless; however, it is not expected that this will generally be the case.

\section{Discussion}

In this section we discuss some of the ways in which the notions of backbones and backdoors can be exploited and gain impact in the field of QSTR.

Satisfiability Modulo Theories (SMT). Over the past years SAT encodings of QCNs have been proposed that are very successful in tackling the hardest of instances, see for example the work in [10]. However, a disadvantage of such encondings is that they generally do not scale well compared to native QSTR tools because they are proportional (to some extent) to the number of variable triples in a given QCN, see for instance the experimental analysis in [10, Section 6.4] and $[9$, Section 5]. The identification of a backdoor in a given QCN for some consistency and parts of a potential backbone, could inspire SMT approaches where the partial backbone along with the rest of the tractable part of the QCN would be dealt with by use of a native QSTR tool, and the hard part, the backdoor, would be treated by an off-the-shelf SAT solver. Further, such combination of theories could occur lazily by allowing each of the involved decision procedures to act as a referee upon the other one at each step of the reasoning process.

Decomposition and Parallelization Techniques. A potential backbone in a QCN can allow for partitioning its constraint graph into simpler to solve instances, perhaps even independently of one another, in parallel; for example, we can imagine the case where a QCN can be viewed as two overlapping instances whose common constraints form a local backbone. In the same vein, exposing a (strong) backdoor in a given QCN with respect to some local consistency, allows for defining search space splitting approaches, i.e., approaches based on dividing the search space of the QCN into disjoint subspaces to be explored in parallel [15].

Adaptive Constraint Propagators. Real-world spatio-temporal datasets that have been studied in the literature tend to be different from synthetic ones, in that they are usually large-scale and composed of a substantial tractable part for even the weakest of local consistencies [18,14,19]; thus, it would be perfectly appropriate to coin the phrase "one consistency does not fit all". The use of backbones and backdoors could help us to understand and quantify in a better manner the (hidden) structural differences between real-world and synthetic datasets, and lead to the definition and implementation of generic algorithms that would adapt themselves to the diverse computational characteristics of the given instance. Furthermore, such notions could be used for establishing heuristics that would guide search in backtracking algorithms more fruitfully. 


\section{References}

1. Alirezaie, M., Längkvist, M., Sioutis, M., Loutfi, A.: Semantic Referee: A NeuralSymbolic Framework for Enhancing Geospatial Semantic Segmentation. Semantic Web (2019), in press.

2. Allen, J.F.: Maintaining Knowledge about Temporal Intervals. Commun. ACM 26, 832-843 (1983)

3. Amaneddine, N., Condotta, J.F., Sioutis, M.: Efficient Approach to Solve the Minimal Labeling Problem of Temporal and Spatial Qualitative Constraints. In: IJCAI (2013)

4. Bhatt, M., Wallgrün, J.O.: Geospatial Narratives and Their Spatio-Temporal Dynamics: Commonsense Reasoning for High-Level Analyses in Geographic Information Systems. ISPRS Int. J. Geo-Information 3, 166-205 (2014)

5. Condotta, J.F., Lecoutre, C.: A Class of ${ }_{f}$-Consistencies for Qualitative Constraint Networks. In: KR (2010)

6. Condotta, J.F., Ligozat, G., Saade, M.: Eligible and Frozen Constraints for Solving Temporal Qualitative Constraint Networks. In: CP (2007)

7. Dylla, F., Lee, J.H., Mossakowski, T., Schneider, T., van Delden, A., van de Ven, J., Wolter, D.: A Survey of Qualitative Spatial and Temporal Calculi: Algebraic and Computational Properties. ACM Comput. Surv. 50, 7:1-7:39 (2017)

8. Dylla, F., Wallgrün, J.O.: Qualitative Spatial Reasoning with Conceptual Neighborhoods for Agent Control. J. Intell. Robotic Syst. 48, 55-78 (2007)

9. Glorian, G., Lagniez, J., Montmirail, V., Sioutis, M.: An Incremental SAT-Based Approach to Reason Efficiently on Qualitative Constraint Networks. In: CP (2018)

10. Huang, J., Li, J.J., Renz, J.: Decomposition and tractability in qualitative spatial and temporal reasoning. Artif. Intell. 195, 140-164 (2013)

11. Krishnaswamy, N., Friedman, S., Pustejovsky, J.: Combining Deep Learning and Qualitative Spatial Reasoning to Learn Complex Structures from Sparse Examples with Noise. In: AAAI (2019)

12. Ligozat, G.: Qualitative Spatial and Temporal Reasoning. Wiley (2013)

13. Ligozat, G., Renz, J.: What Is a Qualitative Calculus? A General Framework. In: PRICAI (2004)

14. Long, Z., Sioutis, M., Li, S.: Efficient Path Consistency Algorithm for Large Qualitative Constraint Networks. In: IJCAI (2016)

15. Martins, R., Manquinho, V.M., Lynce, I.: An overview of parallel SAT solving. Constraints 17, 304-347 (2012)

16. Nebel, B.: Solving Hard Qualitative Temporal Reasoning Problems: Evaluating the Efficiency of Using the ORD-Horn Class. Constraints 1, 175-190 (1997)

17. Renz, J., Ligozat, G.: Weak Composition for Qualitative Spatial and Temporal Reasoning. In: CP (2005)

18. Sioutis, M., Condotta, J., Koubarakis, M.: An Efficient Approach for Tackling Large Real World Qualitative Spatial Networks. Int. J. Artif. Intell. Tools 25, 1-33 (2016)

19. Sioutis, M., Long, Z., Li, S.: Leveraging Variable Elimination for Efficiently Reasoning about Qualitative Constraints. Int. J. Artif. Intell. Tools 27, 1860001 (2018)

20. Sioutis, M., Paparrizou, A., Condotta, J.: Collective Singleton-Based Consistency for Qualitative Constraint Networks: Theory and Practice. Theor. Comput. Sci (2019), in press.

21. Williams, R., Gomes, C.P., Selman, B.: Backdoors To Typical Case Complexity. In: IJCAI (2003) 